\title{
Indocyanine Green Fluorescence-Guided Sentinel Node Biopsy in Breast Cancer Within a North African Population: A Retrospective Study
}

(1) Samir Hidar, (10) Amal Alimi, (10) Abdejlil Khlifi, (1) Selma Chachia, (1) Ons Kaabia, (1) Sassi Bouguizane, (1) Mohamed Bibi, (1) Hédi Khairi

Department of Obstetrics and Gynaecology, F. Hached University Teaching Hospital, Sousse, Tunisia

\begin{abstract}
Objective: Radio isotopes and blue dyes alone or in combination are the most commonly used tracer agents in sentinel node (SN) biopsy for early breast cancer. Recent studies have found fluorescence method using indocyanine green (ICG) as a promising technology with fewer disadvantages.

Materials and Methods: Retrospective analysis of our database that included patients with clinically node-negative breast cancer scheduled for breast surgery and SN biopsy between 2016 and January 2021. Patients who underwent detection using fluorescence-ICG were included in this study.

Results: A total of 47 patients were included. Median age was 50 (range: 24-78) years. Mean tumor size was $3.4 \pm 1.5 \mathrm{~cm}$. All patients received ICG injection and 11 received a combination of ICG and blue dye. Forty-five successful SN identifications with ICG were performed and 99 nodes retrieved. Eleven procedures were undertaken after initial systemic therapy. Twenty-four patients had at least one positive $\mathrm{SN}$ for malignancy. Mean follow up was 29.2 months and no axillary recurrence was noted during the study period.
\end{abstract}

Conclusion: ICG appears to be a feasible and accurate method for SN biopsy with high identification rate. This is the first study of ICG in sentinel node biopsy in a North African population.

Keywords: Breast cancer, indocyanine green, sentinel lymph node biopsy, surgery

Cite this article as: Hidar S, Alimi A, Khlifi A, Chachia S, Kaabia O, Bouguizane S, Bibi M, Khairi H. Indocyanine Green Fluorescence-Guided Sentinel Node Biopsy in Breast Cancer Within a North African Population: A Retrospective Study. Eur J Breast Health 2021; 17(4): 352-355.

\section{Key Points}

- Sentinel node is a standard of care for early breast cancer patients.

- $\quad$ Radio Isotopes alone or combined with blue dye are the most commonly used tracer agents but have several clinical limitations.

- This study is the first to report fluorescence guided sentinel node biopsy in a North African setting and confirms it as a promising technology.

\section{Introduction}

Sentinel node (SN) technique in breast cancer was first describe in 1994 by Giuliano et al. (1) and Krag et al. (2). The aim of the technique is to identify patients who can be spared axillary clearance. Since than the procedure has become a standard approach for breast cancer patients with clinically node-negative disease (3). Radio isotopes (RI) alone or in combination with blue dyes (BD) are the most commonly used tracers. A combination of different tracers is recommended to increase identification rates and decrease false negative rates (3). Despite consistent identification rates higher than 90\% (4), published data (5) report several clinical limitations with these tracers including hypersensitivity, potential radioactivity exposure with high volume activity, the need for specially arranged specimen transportation for pathology, and regular on-site contamination tests. This increases the already high cost further. These drawbacks have led to the development of alternative tracers, such as super-paramagnetic iron oxide nanoparticles (6) and contrast-enhanced ultrasound (7). Numerous cohort studies and clinical trials from Asia $(7,8)$, Europe (9), and the US (10) have suggested the use of indo-cyanine green (ICG) as a promising candidate for tracer in this technique.

To the best of our knowledge, and after an extensive literature search, no study reported the use of ICG fluorescence-guided SN biopsy in breast cancer in North Africa and this study represents the first cohort of ICG fluorescence application within this setting. 


\section{Materials and Methods}

We performed a retrospective review of our prospective, maintained database that included breast cancer planned for SN biopsy at the F. Hached University teaching hospital (Sousse, Tunisia). Data between April 2016 and January 2021 were reviewed. Only non-pregnant or lactating adult female patients without palpable clinical nodes and considered suitable for such procedure at multidisciplinary meetings were included. We excluded from our analysis the first ten cases as these were considered to consitute the "learning curve" (11). A single operator $(\mathrm{SH})$ carried out all the procedures.

Under general anesthesia, $5 \mathrm{~mL}$ at $2.5 \mathrm{mg} / \mathrm{mL}$ of Infracyanine (Laboratoire SERB; France) were injected circumferentially around the areola. Two $\mathrm{mL}$ of patent blue-V dye (Laboratoire Guerbet, Aulney-Sous-Bois, France) were injected in combination in the cohort of patients undergoing the procedure after primary chemotherapy. This was followed by a breast massage to facilitate absorption into the lymph vessels. Then the surgical lights were turned off and images were obtained under near-infrared light using a first-generation near infra-red (NIR) device (12). The course of subcutaneous lymphatic drainage pathway fluorescence was followed up to where it became indistinct as the lymphatics entered deeper in the axilla and an incision was performed at that place. Lymphatic duct identification using the NIR camera allowed localization and removal of the sentinel lymph node(s). Further fluorescent imaging was performed to identify potential residual signal in the axilla and such signal-sites were removed. Following ICG assisted-dissection, blue-stained nodes were excised, if any. After SN biopsy, conservative surgery or mastectomy was performed according to indication and axillary dissection was performed according to international guidelines.

\section{Statistical analysis and ethical approval}

Baseline patient's and tumor characteristics, and identification rate of the SN with ICG and with dye, when used, were recorded. Data were entered and analyzed in Excel then analyzed using PSPP v 1.2.0-3. The continuous variables are presented as mean \pm standard deviation and categorical variables are presented as count and percentages, unless specified.

The study was performed in accordance with the Declaration of Helsinki. All patients provided informed consent following a detailed explanation of the procedures that they may undergo. Institutional ethical review board approval was obtained (F. Hached University teaching Hospital 01/02/2015 and Faculty of Medicine of SousseCEFMS82), the study itself was also retrospectively registered as NCT04879680 (clinicaltrial.gov).

\section{Results}

During the study period, and after exclusion of the first ten cases, 47 patients who underwent SN biopsy using the above-mentioned technique were included. Median age at time of surgery was 50 years (range: $24-78$ years). A total of 45 successful SN procedures were performed (identification rate $=95.7 \%$ ). Median removed nodes per patient was 2 (range: 1-4). In two patients, the procedure failed and they underwent axillary clearance that was, in both cases, pathologically positive.

Thirty-six patients $(76.5 \%)$ received upfront operation while others received primary systemic therapy. ICG-blue dye combination was used for this last group of patients and, in this subgroup, identification rate was $100 \%$ with ICG (11/11) and $81.9 \%$ with blue dye (9/11). Patients and tumor characteristics are described in Table 1.

Twenty-six patients underwent axillary dissection ( 24 positive SN and two failed procedures). The remaining 21 patients with negative SN on frozen section were confirmed thereafter. Importantly, in 11 of the 24 positive $\mathrm{SN}$ cases (45.8\%), SN was a solitary, pathologically positive node on axillary clearance. Median follow-up time was 30 (151) months while median follow-up time for the subgroup of patients with negative SN was 28 (1-51) months and no axillary recurrence was noted in this group during the study period.

\section{Discussion and Conclusion}

With an equivalent overall survival, disease-free survival, and regional control, sentinel lymph node biopsy has become the standard of care for patients with early breast cancer and clinically negative axillary lymph nodes (3) and has gradually replaced axillary dissection indications. Several drawbacks have been reported with conventional methods, such as Isotope and dye, and this has led to the development of new tracers. In the super-paramagnetic iron oxide nanoparticles (6) (SPIO) procedure, magnetic nanoparticles are injected and the $\mathrm{SN}$ is subsequently detected by a hand-held magnetometer. This technique has shown promising results (13) but its major drawback is interference of iron with possible postoperative breast MRI. Contrast-enhanced ultrasound using microbubbles is also a recently developed technique (7). The contrast agent is cheap but the results remain suboptimal and highly operator dependent when compared to other tracers (7). The development and introduction of a fluorescence technique as a new method of $\mathrm{SN}$ detection has allowed a number of these disadvantages to be overcome.

Numerous studies, including clinical trials, evaluating ICG as a tracer for SN have been published worldwide (7-10) but to the best of our understanding, this study represents the first in a North

Table 1. Patients and tumor characteristics, $(n=47)$

\begin{tabular}{lll} 
Mean age, years, (range) & 50.1 & $(24-78)$ \\
Mean body mass index, kg/m² $( \pm$ SD) & 22.7 & $( \pm 3.1)$ \\
Left side tumor & 28 & $(58.6 \%)$ \\
$\begin{array}{l}\text { Tumor size (cm) before surgery } \\
\text { ( } \mathbf{S D})\end{array}$ & 3.1 & $( \pm 1.5)$ \\
Multifocal tumor & 8 & $(17 \%)$ \\
Tumor location & & \\
Upper outer quadrant & 19 & $(41.3 \%)$ \\
Upper inner quadrant & 7 & $(15.2 \%)$ \\
Lower inner quadrant & 9 & $(19.5 \%)$ \\
Lower outer quadrant & 6 & $(10.8 \%)$ \\
Central & 6 & $(13 \%)$ \\
Molecular type & & \\
Luminal A & 7 & $(14.8 \%)$ \\
Luminal B & 7 & $(14.8 \%)$ \\
HER2 enriched & 20 & $(42.5 \%)$ \\
Triple negative & 13 & $(29.7 \%)$ \\
\hline SD: Standard deviation, n: Number & & \\
\hline
\end{tabular}


African population. After a short, technique-specific, learning curve as performed by others (11), we found a $95.7 \%$ identification rate, similar to those reported in the literature, which ranges between $93 \%$ and $100 \%(14,15)$.

The body of information from the current data suggests that using ICG as a tracer allows real-time visualization of lymphatic channels, provides a high identification rate with a low dose of tracer and has an excellent safety profile in clinical use (16). Two recent, comprehensive meta-analyses $(14,15)$ addressed the question of whether ICG can act as a better tracer agent compared with conventional techniques. ICG alone is a better tracer agent compared with $\mathrm{BD}$ and/or $\mathrm{RI}$ alone and is not inferior compared to $\mathrm{BD}$ and $\mathrm{RI}$ in combination $(14,15)$. Yin et al. (15) concluded that ICG was a suitable alternative to traditional tracers to detect $\mathrm{SN}$ in patients with breast cancer while Kedrzycki et al. (14) recommended that "hospitals using RI and or BD could consider changing their practice to ICG”. Some studies suggested that the rapid spread of ICG and high sensitivity of fluorescence detection devices lead to a greater number of dissected SN with ICG fluorescence when compared to RI and/or blue dye. (17-19). We retrieved a mean of 2.2 nodes per procedure and our data do not support this but our sample size was limited.

Age over 60 and high body mass index (BMI) were suggested to negatively affect identification rate (14) but results of the different studies are conflicting. In our study, both cases in whom identification failed were less than 60 years and both had a BMI of 25 . None of these two patients received neoadjuvant chemotherapy and both had positive macrometastatic nodes at axillary clearance. Whether node invasion by lymphatic channel obstruction is a risk factor for identification failure is a matter of debate and needs further studies.

Eleven patients in our study group received neoadjuvant chemotherapy prior to SN. They received combined ICG-blue dye technique and SN localization was successful with ICG in all patients in this subgroup. This is in accordance with recent studies that show promising results of this tracer combination after neoadjuvant chemotherapy with an identification rate ranging from $83 \%$ to $100 \%(20-22)$ and very low false-negative rates (22).

Cost-effectiveness of ICG is a matter for debate. Near Infrared camera devices are expensive (basic models start at 19,000 Euros) but Indocyanine green tracer prices range from 18 Euros in India (Aurogreen $^{\circledast}$ ) to 124 Euros for Infracyanine ${ }^{\circledast}$ that we used in our study. Once opened the vial should be used within eight hours, and the amount is sufficient for two procedures. Thus in high volume units the procedure will be more cost effective than in smaller units and may even compare with Patent Blue ${ }^{\circledast}$.

Limitations of our study include selection bias, absence of direct comparison with conventional tracer, and absence of systematic axillary clearance for the entire cohort to assess the false-negative rate. However, in our study, $45.8 \%$ of patients had a solitary sentinel node with negative axillary clearance. This is a clear positive quality indicator and in accordance with previously published data (9).

No recurrence occurred in our study, but the median follow-up was relatively short (30 months). A recent long-term follow-up study by Wang et al. (22) confirmed that the ICG-BD combination was safe. In 687 early breast cancer patients who underwent the procedure with histologically negative $\mathrm{SN}, 0.64 \%$ recurred after a median follow-up
This study is the first cohort in whom ICG fluorescence was used for $\mathrm{SN}$ in breast cancer in a North African setting and includes a reasonable sized cohort with a single operator. The high identification rate of the technique in the present study is in accordance with recent comprehensive meta-analysis and confirms its potential to become the standard of care in this indication.

Ethics Committee Approval: Institutional ethical review board approval was obtained (F. Hached University teaching Hospital 01/02/2015 and Faculty of Medicine of Sousse- CEFMS82), the study itself was also retrospectively registered as NCT04879680 (clinicaltrial.gov).

Informed Consent: All patients provided informed consent following a detailed explanation of the procedures that they may undergo.

Peer-review: Externally peer-reviewed.

\section{Authorship Contributions}

Surgical and Medical Practices: S.H., Concept: S.H., A.A., A.K., S.C., O.K., S.B., M.B., H.K., Design: S.H., A.A., A.K., S.C., O.K., S.B., M.B., H.K., Data Collections and/or Processing: A.A., A.K., S.C., O.K., Analysis and/or Interpretation: S.H., A.A., A.K., S.C., O.K., S.B., M.B., H.K., Literature Search: A.A., A.K., S.C., O.K., S.B., M.B., H.K., Writing: S.H., A.A., A.K., S.C., O.K., S.B., M.B., H.K.,

Conflict of Interest: The authors certify that there is no conflict of interest with any financial organization regarding the material discussed in the manuscript.

Financial Disclosure: The authors declare that this study received no financial support.

\section{References}

1. Giuliano AE, Kirgan DM, Guenther JM, Morton DL. Lymphatic mapping and sentinel lymphadenectomy for breast cancer. Ann Surg 1994; 220: 391-398; discussion 398-401. (PMID: 8092905) [Crossref]

2. Krag DN, Weaver DL, Alex JC, Fairbank JT. Surgical resection and radiolocalization of the sentinel lymph node in breast cancer using a gamma probe. Surg Oncol 1993; 2: 335-339; discussion 340. (PMID: 8130940.) [Crossref]

3. Maguire A, Brogi E. Sentinel lymph nodes for breast carcinoma: an update on current practice. Histopathology 2016; 68: 152-167. (PMID: 26768036). [Crossref]

4. Lyman GH, Somerfield MR, Giuliano AE. Sentinel lymph node biopsy for patients with early-stage breast cancer: 2016 American Society of Clinical Oncology clinical practice guideline update summary. J Oncol Pract 2017; 13: 196-198. (PMID: 28118104) [Crossref]

5. de Kanter AY, Arends PP, Eggermont AM, Wiggers T. Radiation protection for the sentinel node procedure in breast cancer. Eur J Surg Oncol 2003; 29: 396-399. (PMID: 12711297) [Crossref]

6. Karakatsanis A, Olofsson H, Stålberg P, Bergkvist L, Abdsaleh S, Wärnberg F. Simplifying logistics and avoiding the unnecessary in patients with breast cancer undergoing sentinel node biopsy. A prospective feasibility trial of the preoperative injection of super paramagnetic iron oxide nanoparticles. Scand J Surg 2018; 107: 130-137. (PMID: 29132268) [Crossref]

7. Cox K, Dineen N, Taylor-Phillips S, Sharma N, Harper-Wynne C, Allen D, et al. Enhanced axillary assessment using intradermally injected microbubbles and contrast-enhanced ultrasound (CEUS) before neoadjuvant systemic therapy (NACT) identifies axillary disease missed by conventional B-mode ultrasound that may be clinically relevant. Breast Cancer Res Treat 2021; 185: 413-422. (PMID: 33029707) [Crossref] 
8. Sugie T, Kinoshita T, Masuda N, Sawada T, Yamauchi A, Kuroi K, et al. Evaluation of the clinical utility of the ICG fluorescence method compared with the radioisotope method for sentinel lymph node biopsy in breast cancer. Ann Surg Oncol 2016; 23: 44-50. (PMID: 26275781) [Crossref]

9. Hirche C, Mohr Z, Kneif S, Murawa D, Hünerbein M. High rate of solitary sentinel node metastases identification by fluorescence-guided lymphatic imaging in breast cancer. J Surg Oncol 2012; 105: 162-166. (PMID: 21882198) [Crossref]

10. Troyan SL, Kianzad V, Gibbs-Strauss SL, Gioux S, Matsui A, Oketokoun $\mathrm{R}$, et al. The FLARE intraoperative near-infrared fluorescence imaging system: a first-in-human clinical trial in breast cancer sentinel lymph node mapping. Ann Surg Oncol 2009; 16: 2943-2952. (PMID: 19582506) [Crossref]

11. He K, Chi C, Kou D, Huang W, Wu J, Wang Y, et al. Comparison between the indocyanine green fluorescence and blue dye methods for sentinel lymph node biopsy using novel fluorescence image-guided resection equipment in different types of hospitals. Transl Res 2016; 178: 74-80. (PMID: 27497181) [Crossref]

12. Kitai T, Inomoto T, Miwa M, Shikayama T. Fluorescence navigation with indocyanine green for detecting sentinel lymph nodes in breast cancer. Breast Cancer 2005; 12: 211-215. (PMID: 16110291) [Crossref]

13. Zada A, Peek MC, Ahmed M, Anninga B, Baker R, Kusakabe M, et al. Meta-analysis of sentinel lymph node biopsy in breast cancer using the magnetic technique. Br J Surg 2016; 103: 1409-1419. (PMID: 27611729). [Crossref]

14. Kedrzycki MS, Leiloglou M, Ashrafian H, Jiwa N, Thiruchelvam PTR, Elson DS, et al. Meta-analysis comparing fluorescence imaging with radioisotope and blue dye-guided sentinel node identification for breast cancer surgery. Ann Surg Oncol 2021; 28: 3738-3748. [Crossref]

15. Yin R, Ding LY, Wei QZ, Zhou Y, Tang GY, Zhu X. Comparisons of ICG-fluorescence with conventional tracers in sentinel lymph node biopsy for patients with early-stage breast cancer: A meta-analysis. Oncol Lett 2021; 21: 114. (PMID: 33376546) [Crossref]
16. Stanescu-Segall D, Jackson TL. Vital staining with indocyanine green: a review of the clinical and experimental studies relating to safety. Eye (Lond) 2009; 23: 504-518. (PMID: 18670454) [Crossref]

17. Goonawardena J, Yong C, Law M. Use of indocyanine green fluorescence compared to radioisotope for sentinel lymph node biopsy in early-stage breast cancer: systematic review and meta-analysis. Am J Surg 2020; 220: 665-676. (PMID: 32115177) [Crossref]

18. Tong M, Guo W, Gao W. Use of fluorescence imaging in combination with patent blue dye versus patent blue dye alone in sentinel lymph node biopsy in breast cancer. J Breast Cancer. 2014; 17: 250-255. (PMID: 25320623) [Crossref]

19. Takao Y, Toh U, Sakurai S, Saku S, Takenaka M, Akashi M, et al. [Triple-tracer technique of sentinel lymph node biopsy using blue dye plus radioisotope combined with real-time indocyanine green (ICG) fluorescence imaging procedures for patients with breast cancer treated with neoadjuvant chemotherapy] [Article in Japanese]. Gan To Kagaku Ryoho 2020; 47: 1225-1227. (PMID: 32829360) [Crossref]

20. Agrawal SK, Hashlamoun I, Karki B, Sharma A, Arun I, Ahmed R. Diagnostic performance of indocyanine green plus methylene blue versus radioisotope plus methylene blue dye method for sentinel lymph node biopsy in node-negative early breast cancer. JCO Glob Oncol 2020; 6: 1225-1231. (PMID: 32749861) [Crossref]

21. Chirappapha P, Chatmongkonwat T, Lertsithichai P, Pipatsakulroj W, Sritara C, Sukarayothin T. Sentinel lymph node biopsy after neoadjuvant treatment of breast cancer using blue dye, radioisotope, and indocyanine green: prospective cohort study. Ann Med Surg (Lond) 2020; 59: 156160. (PMID: 33072308) [Crossref]

22. Wang C, Tong F, Cao Y, Liu P, Zhou B, Liu H, et al. Long-term follow-up results of fluorescence and blue dye guided sentinel lymph node biopsy in early breast cancer. Breast Cancer Res Treat. 2021; 188: 361-368. (PMID: 33761081) [Crossref] 